\title{
Design and Deployment of Vehicular Internet of Things for Smart City Applications
}

\author{
Evariste Twahirwa $^{1, *,+(\mathbb{D})}$, James Rwigema ${ }^{1,+}$ and Raja Datta ${ }^{2}$ (D) \\ 1 African Center of Excellence in Internet of Things, College of Science \& Technology, University of Rwanda, \\ Kigali 3900, Rwanda; j.rwigema@ur.ac.rw \\ 2 Department of Electronics \& Electrical Communication Engineering, Indian Institute of Technology, \\ Kharagpur 721302, India; rajadatta@ece.iitkgp.ac.in \\ * Correspondence: evatwa@yahoo.com or e.twahirwa@ur.ac.rw; Tel.: +250-788467863 \\ + These authors contributed equally to this work.
}

Citation: Evariste, T.; Rwigema, J.; Datta, R. Design and Deployment of Vehicular Internet of Things for Smart City Applications. Sustainability 2022, 14, 176. https:// doi.org/10.3390/su14010176

Academic Editors: Marija

Burinskienè and Rasa

Ušpalytè-Vitkuniene

Received: 3 November 2021

Accepted: 9 December 2021

Published: 24 December 2021

Publisher's Note: MDPI stays neutral with regard to jurisdictional claims in published maps and institutional affiliations.

Copyright: (C) 2021 by the authors. Licensee MDPI, Basel, Switzerland. This article is an open access article distributed under the terms and conditions of the Creative Commons Attribution (CC BY) license (https:/ / creativecommons.org/licenses/by/ $4.0 /)$.

\begin{abstract}
A novel computing paradigm, called the Internet of things emerged a few years ago. IoT is materialized by connecting both real and digital worlds together. The deployment of IoT in vehicular networks has introduced a new vehicular communication technology-themed vehicular internet of things (V-IoT). With the introduction of miniaturized sensors and actuators, V-IoT has demonstrated the ability to improve the level of urban transport systems through the development and deployment of low-cost but powerful technologies which seamlessly upgrade the level of smart transportation in urban environments. In this research article, we have presented the features of V-IoT that encompass both the benefits and potential challenges of the technology. Low-cost IoT prototypes have been built and tested for numerous functions in vehicular environments. The monitored parameters include air, road conditions such as traffics flow sizes, air quality, weather parameters, and signal status in terms of Received signal strength indicator, and Signal noise ratio for both road and intra-vehicular environments. Devices are implemented at every IoT architectural layer and tested on a web-based IoT front-end application using different protocols like LoRaWAN. Two LoRa sensors have been deployed in the public bus to monitor some of the mentioned parameters on a real-time basis and historical data could be retrieved through the developed web-based dashboard. Simplistic algorithms are implemented for both real-time and historical data demonstration.
\end{abstract}

Keywords: vehicular internet of things; internet of things; smart city

\section{Introduction}

In recent years, there has been a steady growth in Internet of Things (IoT), a novel paradigm that is being materialized by integrating physical objects [1-3]. The services the technology exhibits to numerous sectors like Smart city, Smart Agriculture, Smart Manufacturing, and others are understandable. For instance, Internet of battlefield things, Internet of medical things, Internet of animal things, and others have been introduced [4]. IoT-based smart city implementation needs a massive deployment of IoT devices, the associated big data and information requires cloud computing to handle storage and complexity computational issues. The intelligent data transmission algorithms based on data aggregation are very vital, this avoids bandwidth misuse in re-transmission of same data streams [5]. The role of IoT is to equip physical objects with sensing, computing, and communicating capabilities so that they can interact with each other over an established network and following the user defined rules [6]. In this work, we define the vehicular Internet of things (V-IoT) as a vehicular network where IoT devices, systems, and frameworks are deployed to monitor vehicular environments for numerous purposes such as road contexts, vehicle's position, weather and environment monitoring, and so forth. The V-IoT may as well be defined as a form of vehicular network where communicating entities are mobile and/or stationary vehicles supplemented by the deployed IoT technologies, the 
paradigm has been realized from vehicular Ad Hoc Network that has been in existence for several decades and internet of things [7,8]. V-IoT has got a number of applications related to smart cty these include (i) road context-sensing, (ii) vehicle tracking, (iii) monitoring of intra-vehicular environments, traffic flow monitoring,etc. V-IoT is expected to ensure vehicular connections such as vehicle to road, vehicle to vehicle, vehicle to infrastructure, and possibly vehicle to everything (V2X) and improve the smart city applications soon after their deployment. Globally, IoT is considered as the major smart city enabler that facilitates the implementation numerous smart city applications [9-12].

In this article, we implement different IoT-based solutions that would facilitate urban transport systems and fix the potential issues common in vehicular networks. Road context is lively monitored and the collected information could easily be transmitted to the endusers that are truly concerned. Drivers are able to view the traffic sizes in their directions which would assist them to take alternative routes in case of traffic congestion in their. V-IoT has got special features, and architecture that makes it unique compared to other IoT networks; these features are explained here below:

\subsection{V-IoT Generalized Architecture}

In this work we introduce a vehicular IoT architecture that is based on the three-tier layered IoT architecture. The three layers are Perception/things or physical layer at the bottom, Network (middle) Layer, and Application (top) layer.

- $\quad$ V-IoT Perception Layer

The fist layer in a V-IoT architecture the perception layer is found at the bottom of the architecture. It comprises of different vehicular sensors that gather and collect data from the vehicle itself and its environment. In our developed systems, we have implement a multi-sensing IoT unit and deployed it at the perception layer to collect relevant information that are important for seamless driving in the case study area. The unit comprises of web-cam, GPS, air quality, weather monitoring sensing modules, and buzzers to actuate alarms in case of abnormal level of the monitored parameters.

- V-IoT Network Layer

The network layer interconnects the smart devices at the perception to the dedicated network infrastructure like network devices, gateways, severs, needed for data transmission and processing. The network layer links the things layer with application layer. The network infrastructures such as gateways, application servers, are all configured at the network layer of the IoT architecture to converge all collected data from the vehicular entities and environments to the dedicated storage and processing systems for further analysis and presentation.

- V-IoT Application Layer

The application layer is the top-most layer that extends the V-IoT architecture to the end-users that are truly concerned. Mobile applications, machine learning applications, data analysis and visualization are found at the application layer. Users could interact with the IoT system through the developed application programming interfaces (APIs). Several Web based front-end IoT platforms are available for specific applications configuration and customization. Sensors, gateways, edge, and cloud configuration could be carried out at this layer. Novel machine learning and artificial algorithms are also implemented based on the needs of end-users.

\subsection{Benefits of V-IoT}

In this part, we narrate the potential benefits of IoT-supported vehicular networks based on the aspects of smart city. V-IoT derives its challenges and benefits from its main features and how generally it operates. Some of its features stand as potential benefits; while others exist as the potential challenges that hinder the adoption and operation of V-IoT.

(i) Wide coverage

In urban environments, vehicles are ubiquitous in nature, this creates a pervasive 
communication in an ad hoc manner that covers large areas in urban areas including highways [13].

(ii) Known mobility routes

Urban roads are fixed, some vehicles pass in specific routes with known start and end points, this creates known routes. Moreover, vehicles equipped with GPS modules could capture road travel history information, such information could be used for traffics flow management, vehicular location monitoring, and so forth.

(iii) Alternative energy sources

V-IoT electronics could be powered by the engines of vehicles when they are moving. When vehicles are parked, several alternative energy sources like rechargeable batteries, suitable solar panels can power the V-IoT electronics. The emerging of energy harvesting technologies promises stable energy efficient vehicular networks [14].

(iv) Edge and cloud integration

The possibility to integrate edge and cloud computing in vehicular networks creates important distributed network system required by urban computation tasks. Computation offloading, and resource allocation is an important aspect that enhances cloud and edge in vehicular network systems [15].

\subsection{Open Issues in V-IoT}

(i) Initial high investment cost

Even-though IoT allows low-cost system development, deployment in sparse areas like cities for full coverage incurs high initial investment cost. Some works have identified vehicles themselves as temporal communication infrastructure [16].

(ii) High speedsz

Compared to ordinary mobile Ad Hoc networks, V-IoT nodes exist in a more dynamic environment. This leads to network topological changes in a more often fashion. Research works have been conducted related to routing in vehicular networks. But efforts are required in the designing algorithms and routing protocols that govern such dynamism observed in the vehicular networks [17].

(iii) Communication heterogeneity

The heterogeneity in communication technologies is also a challenging factor. V-IoT ecosystem brings together diverse entities that like vehicles, passengers, sensors, etc. Different entities employ different communication protocols, and generate different data types. Harmonizing such diversity becomes a research issue.

(iv) Context aware representation

Context-awareness representation in V-IoT, and other vehicular-enabled networks is an issue. In a smart transport environment, all is fine if participating entities are aware of their surroundings, particularly vehicles should be cognizant of their environmental factors, especially those of greater interest like driving behavior, assistance, parking, etc., [18].

(v) Large data volumes \& handling

V-IoT system considers heterogeneous devices that generate large and multidimensional large volumes of data from multiple vehicles that require advanced knowledge and expertise in big data for storage, processing, and analysis.

\section{Related Work}

In recent years there was an interesting trend in the development of internet of things. Particularly, the adoption of IoT in vehicular envronments is not left behind. The revolution of automobile industry to produce autonomous vehicles is a global concern. With the fast development of internet of things, and its frameworks, IoT is expected to transform the automobile industry. Likewise, the development of automobile is as well expected to be the next frontiers of IoT [19]. A distributed real-time IoT for self-driving vehicles is presented in [20], the research in this article learns the impact of delay-accuracy in a 
real-time distributed IoT systems for self-driving vehicles. The authors assume a smart traffic control framework that enables both self-driving vehicles and roadside infrastructure to enhance the efficiency of the intersection to ensure reduced environmental effects due to fuel consumption. In [21], authors introduce a new theme called Quality of IoT-experience within autonomous vehicles. Apart from the concerned end-users, the quality of evaluation involves the machines that are part of the ecosystem. The novel architecture presented in this work considers the quality of data, quality of network, quality of context, to ensure the whole quality IoT in the autonomous vehicles. The confluence of artificial intelligence and IoT is considered to transform the vehicles into a fully smart autonomous vehicle that will provide a support between the real-world and digital world for the next industry 4.0 [22] Whereas the work of [23]presents the integration challenges of Intelligent transport systems, connected vehicles, cloud computing technology, and internet of things. The article discusses some integration challenges that must be dealt with to solve ITS related issues like fuel costs, high carbon emissions, traffic congestions, and so forth.

Authors in [24] study a trust management in a decentralized vehicular internet of things environment. The trust management presented in this work involves the trust management about the message exchange and trust about the node sending the message. The study suggests a fuzzy logic scheme that evaluates the trust of one-hop neighboring nodes to ensure cooperativeness, honestness, and responsibility issues. A Q-learning techniques is introduced to evaluate the trust between other nodes and a trustor node. A trust analysis approach is proposed in [25] for communication in vehicular entities using IoT to ensure greenness of cities. In [26], a smart vehicular framework is implemented based on the internet of things. The proposed IoT system has two major applications, firstly the system detects road irregularities, and secondly the system provides smart vehicular parking system in the urban areas. The road irregularity monitoring component comprises of various IoT sensors and modules including accelerometer, GPS module, a microcontroller unit, a wireless module based on 802.15 .4 protocol, and a cloud service. Whereas the smart vehicular parking component consists of 3D accelerometer, an audio and video interface, GPS, a two-way wireless interface, an on-board processing unit. The system is flexible to allow removal of any module, or could be scaled up by adding extra sensors and modules. The Vehicular IoT sensors are wirelessly interfaced using ZigBee protocol. The work of authors in [27] the previous advances, and open issues have been presented for federated learning of vehicular internet of things. The paper discusses the use of federated learning in V-IoT environments, existing works, technical challenges and possible solutions are discussed as well.

While research works have been conducted related to the vehicular IoT, this new technology still needs improvement in the implementation as well as the general body of knowledge. In this work we discuss the existing benefits of the V-IoT as well as its potential challenges. We build on the existing challenges and attempt to implement technological solutions that attempt to overcome some of these challenges. Multi-sensing IoT-based unit is implemented and tested on numerous vehicular applications. The built system has several functions like road conditions monitoring (traffic flows, road maintenance activities), road descriptions presentation to the drivers, passengers, and other road users. Also an air quality monitoring components is included on the system to ensure pollutionfree urban environments. The existing infrastructure that connect vehicular entities in urban environments require high investment cost related to procurement,deployment, and maintenance. Developing and deployment of IoT low-cost systems would serve as a stepping stone towards finding solutions related to the cost problems.

\section{Materials, Methods, and Case-Study Area}

This study introduces and architectural design for vehicular sensing using IoT Figure 1. Sensing nodes are deployed alongside roads from the case-study areas to collect numerous contextual data that s useful for road users. This include road air pollution, weather parameters such temperature and humidity, road conditions like traffic size, maintenance 
activities and so on. Furthermore, sensors are deployed in vehicular micro-environments to monitor the internal environments of vehicles, especially the public transport buses. All sensor data collected from the target environments are converged to a gateway which links the sensing nodes to the internet. Application servers and programming interfaces are configured to manage storage of our applications for data processing, retrieval, and display. Through different device types, users could query the system for data visualization through an intuitive web-based dashboard.

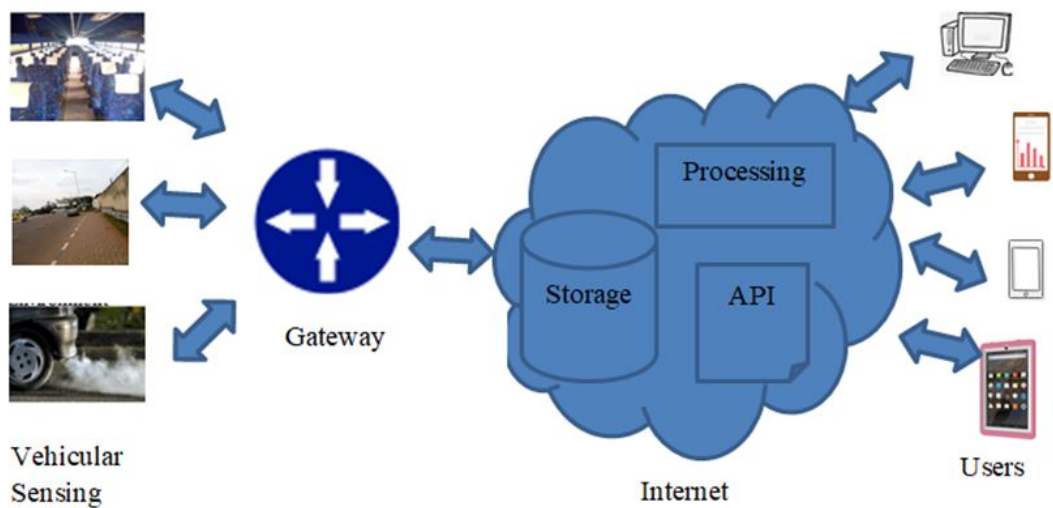

Figure 1. Proposed System.

In the following Figure $2 \mathrm{a}$, we demonstrate the route of the bus in which the experimentation was conducted. Two Lora compliant sensors were deployed in the bus to monitor the level of carbon dioxide and particulate matter 2.5. Table 1 shows the characteristics of the sensors utilized for the gateway testing. The route is chosen based on the position of the Lora gateway. Interestingly from the start to the destination of the chosen route, the sensors on-board are connected to the gateway. Apart from the two key pollutants being monitored, both sensors have got weather and signal status monitoring capacities. The two LoRa compliant sensors have been employed to monitor the saturation of the monitored parameters from the target environments. The sensors were deployed in the bus whose travel routes fall into the coverage of the gateway. Real-time information is retrieved through an intuitive web-based dashboard. The bus route is extracted from the case study area Figure $2 b$. The map of main roads is extracted from openStreet Map to observe how many roads are present and how many of our devices could be deployed for the entire area coverage. We have employed the simulation of urban mobility (SUMO)-simulator to model the macroscopic roads from the case study area. The simulator helps us to understand the nature of the target area regarding the signal interference. All urban fixtures are observed and the geographical locations estimated; these include roads, water-ways, buildings, and other urban features. From the case-study area, roads are represented by the edges of the map, whereas the buildings are represented by polygons, nodes represent the road intersections. Even-though the case study area encompasses a mixture of main and residential routes that connect residential areas to the main roads, our target will be monitoring the main roads that experience heavy traffic volumes on a daily basis. Main routes are indicated by the major edges from the case study area map.

Table 1. Sensor Properties

\begin{tabular}{cc}
\hline Parameter Symbol/Name & Parameter Description \\
\hline Sensor Module & long range wide area network (LORAWAN) \\
Transmission Range & Upto $20 \mathrm{~km}$ \\
Frequency type & License-free bands \\
TCP / IP protocol & Message Queuing Telemetry Transport (MQTT) \\
Transmission Power & $100 \mathrm{~mW}$ \\
\hline
\end{tabular}




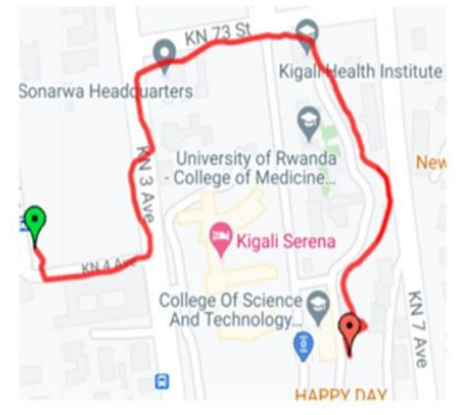

(a) Route of the monitored vehicle

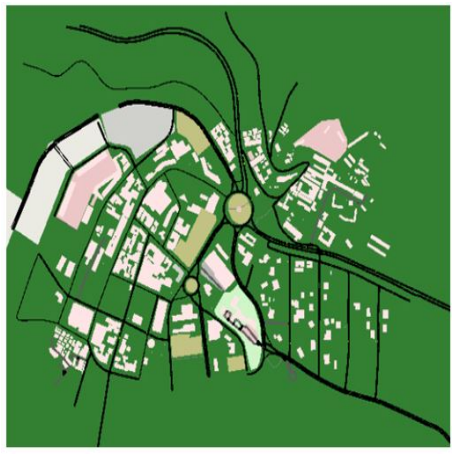

(b) Routes in the case study area

Figure 2. The Monitored bus route and case study area.

\subsection{V-IoT Based Multi-Sensing Test-Bed Components}

The IoT-based V-IoT multifunctional test-bed has been built and deployed for road contextual data collection. Figure $3 a, b$ show the schematic circuit design and the prototype of the road contextual data collection multi-sensing IoT unit. The prototype is constructed using a system-on-chip WiFi module that enables data transmission to the end-users. Our test-bed is powered by a battery with enough power rating (12V-7AH). The system perfectly monitors different road contextual conditions for both safety and non-safety conditions for vehicular use. We use a printed circuit board to mount our control unit and the IoT sensors. The road contextual data provides both safety and non-safety applications, such as, alerting drivers, passengers, and other traffic monitoring personnel on routes with heavy traffic volumes in urban scenarios. We explain each service rendered by our prototype and the component that handles it in the following paragraphs:

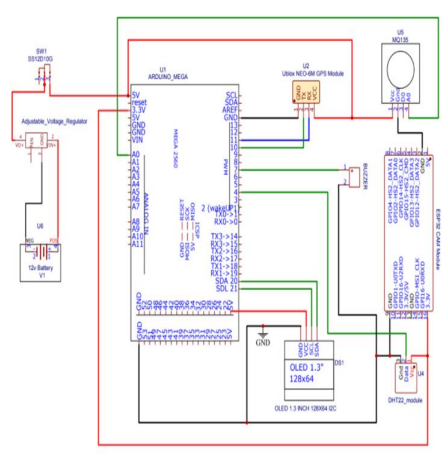

(a) Test-bed Circuit design

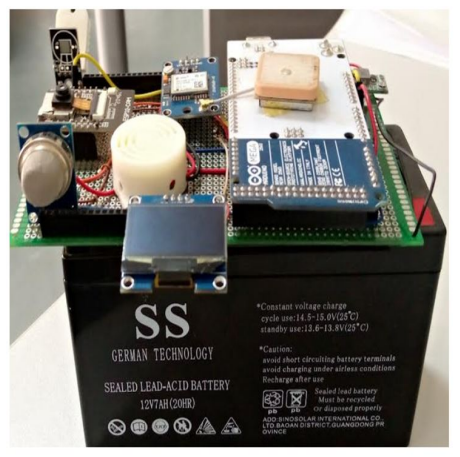

(b) Multsensing test-bed

Figure 3. Circuit design and Prototype multi-sensing unit for V-IoT.

(i) Road condition monitoring (RCM)

An ESP-32 camera module that supports image and video upload is employed to monitor road conditions. The camera module is embedded in ESP32, a low-cost low-power system on chip micro-controller unit with integrated Wi-Fi and dual-mode Bluetooth. Heavy traffic flow routes, road maintenance activities and other road situation contextual information would be detected and presented to drivers and traffic monitoring officials for quick decisions on whether to use or avoid a particular road. Additionally, the developed system can be used to monitor the internal environments of vehicles, especially public buses for seats availability, safety, and other intra-buses conditions. In this section, we show the testing of the developed IoT prototypes mainly for vehicular applications. In Figure $4 \mathrm{a}$ we show the monitoring of urban roads from the case-study area. We use the prototype to stream the condition of one of the roads in the case-study area. The device was deployed at 
a height of $5 \mathrm{M}$ from the ground to monitor a reasonable length of the road. Road conditions could be monitored and transmitted in real-time.

The camera module's field of view (FOV) gives broader focus of the area and will enable road users to monitor the current road conditions which would support in driving decisions. The end-users are given the webcam module IP which they use to connect to the road being monitored. In Figure $4 \mathrm{~b}$ we show the monitoring of intra-vehicular environments using an IoT-based webcam module included on our prototype. This process would assist the passengers waiting at the next bus stop to have knowledge about the availability of the seats in the bus. Likewise the same kit deployed at the bus stop would enable drivers of the public transport buses have an idea of the length of the passengers queue at the next closer bus stops. This will solve the issues of passengers overstaying at the bus stops waiting for full buses. Once their trips are delaying commuters would use such information to look for alternatives if several coming buses have no free seats; for instance they can book motorbikes, tax, and other quick transport facilities. This will serve as a solution to the irritation of passengers that spend more time on road waiting the bus.

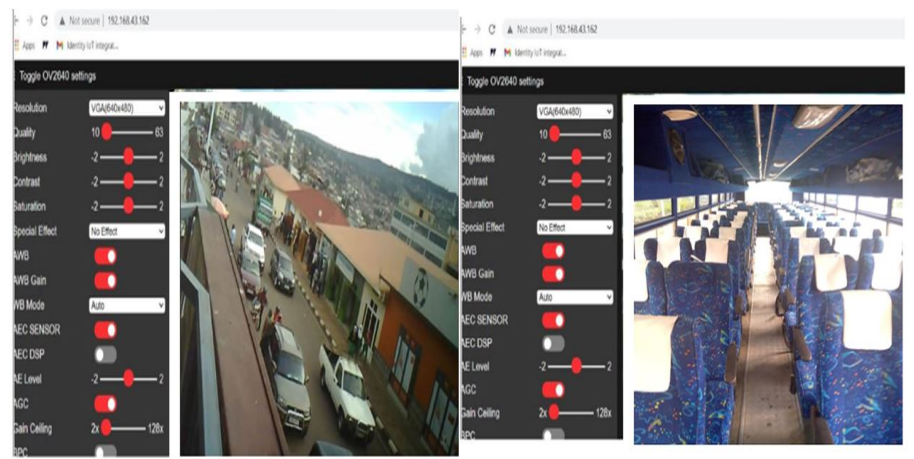

(a) Traffic monitoring

(b) Intra-vehicular monitoring

Figure 4. Traffic and intra-vehicular environments monitoring.

(ii) Air quality monitoring (AQM)

MQ135 sensor module is used to implement the AQM service. AQM monitors a range of air pollutants including $\mathrm{CO}, \mathrm{CO}_{2}$, and $\mathrm{NH} 4$. In reality, a highly polluted road may be a sign of heavy traffic flows. Using such information, the system may advice the drivers to avoid such routes and find alternative to keep the pollution of the area under control. Also, these paths may be avoided for the passengers having cardiovascular, respiratory and other lungs related problems. Two Lora-enabled sensors are employed as well to monitor $\mathrm{CO}_{2}$ and PM2.5 from intra-vehicular environments in real-time basis.

(iii) Road Temperature and humidity monitoring

DHT22 sensor is used to monitor the road environment temperature and humidity. The sensor comprises of two parts, a capacitive humidity sensor and a thermistor. The sensor is also equipped with a built-in chip that converts analog to digital. This gives out digital signal with temperature and humidity values which are compatible with any micro-controller. Figure $5 \mathrm{a}, \mathrm{b}$ demonstrate the temperature and humidity monitoring using the prototype

(iv) Alarming

Buzzers are installed for sound alarms to beep when the value of a parameter being measured becomes higher than a fixed threshold. For instance if the level of $\mathrm{CO}_{2}, \mathrm{Co}$, temperature and humidity exceeds the safety allowable limits to human lives, the buzzer sets an alarm the end-users that are truly concerned will take appropriate mitigation measures. Moreover, this would serve as a stepping stone for environment monitoring 
personnel to find out suitable measures towards smart and clean roads environment for a smart city.

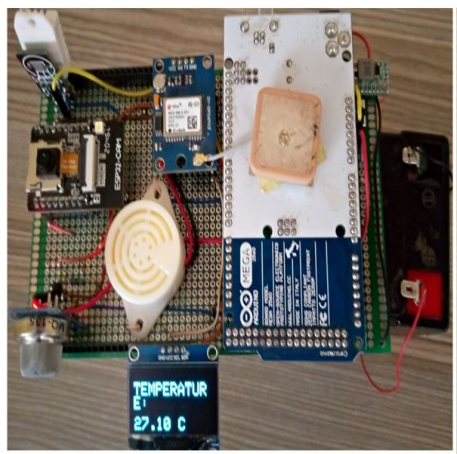

(a) Temperature monitoring

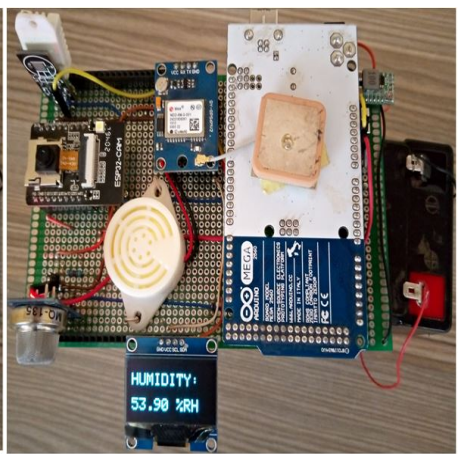

(b) Humidity

Figure 5. Temperature and Humidity monitoring.

(v) Processing

The MCU is the heart of our prototype; its main duty is to manage the functions and interfacing of all other modules. An arduino mega 2560 micro-controller board is employed. The MCU is based on the ATMega2560, and AVR RISC processor that execute powerful instructions in a single clock cycle. This enables the trade-offs between power utilization and processing speed. It should be noted that additional important modules can be introduced based on the users' needs. For instance, RSUs may be made to monitor the driving pattern of the vehicles for controlling the traffic jams and also road accidents avoidance.

\section{(vi) Road description}

Our roadside unit has road name presentation application, which includes the road, street, and avenue details that are displayed to the users. Novice drivers and city visitors will get the mentioned details for help during the driving duration. Our system compliments any existing system that may be available by presenting the city-district-route number information to the road users. OLED-based display is used to implement the receiver and display of the device to show road description, speed limit, weather parameters. Figure $6 \mathrm{a}, \mathrm{b}$ show the existing and implemented road-name display.

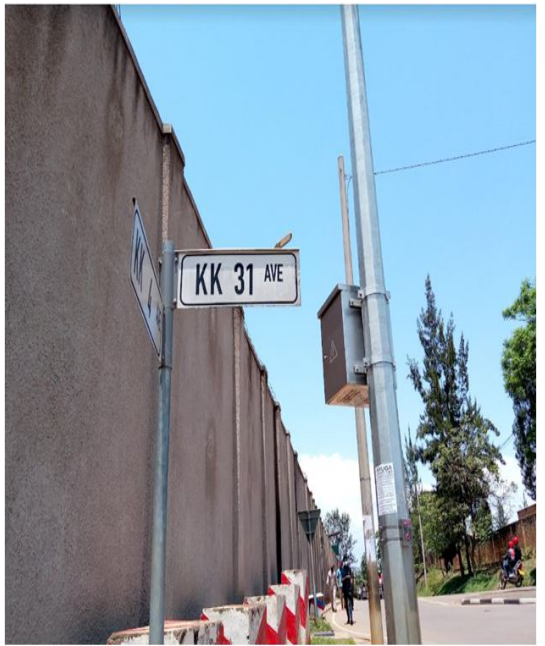

(a) Existing road-name display

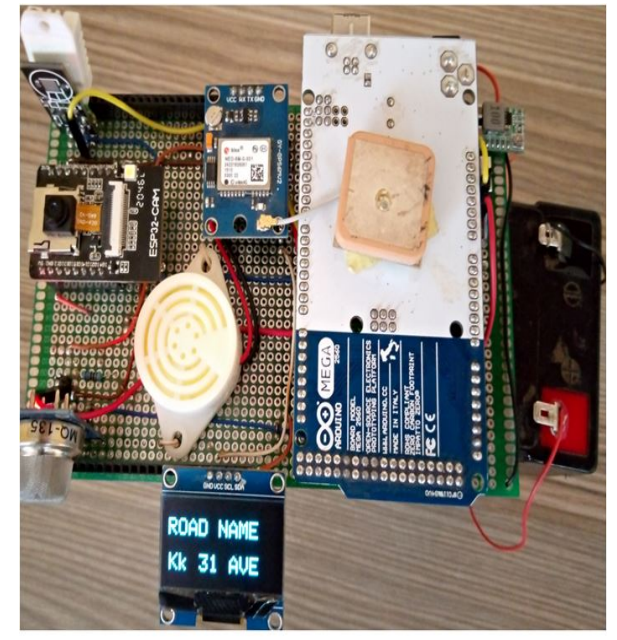

(b) implemented road-name demonstration

Figure 6. Existing and implemented road-name demonstration.

(vii) Allowable speed limit display (ASLD) 
In intelligent transport system, presenting contextual data regarding allowable vehicle speed levels at some points of interest is very crucial. The ASLD service continuously streams the allowable speed limits of specific roads to the drivers using WiFi. The service complements the static speed limit road signs currently in place of the case study area. Though static road-signs exist in the case study area that demonstrate allowable speed limits, it is worthwhile very crucial to constantly display the allowable speed limits to drivers especially in risky roads or to the drivers of some special vehicles such as loaded trucks. Figure $7 \mathrm{a}, \mathrm{b}$ represents the existing and an implemented complementary speed limit display.

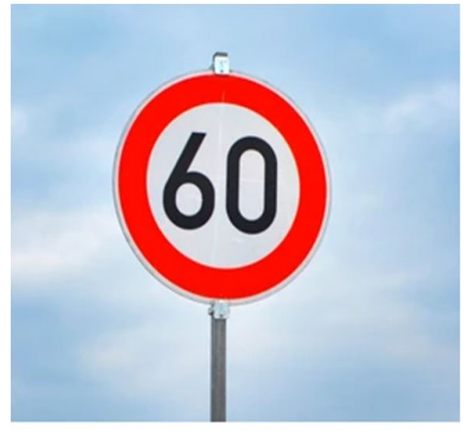

(a) Existing speed limit show

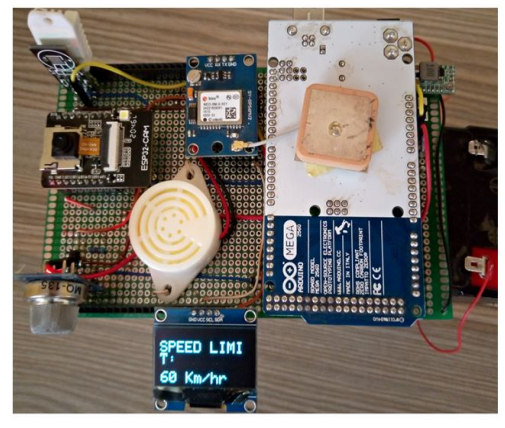

(b) implemented speed limit display

Figure 7. Existing and implemented speed limit display system.

(viii) Position contextual-data collection (PCC)

A global positioning system module is employed to present the positions of interest along the roads in the target area. GY-GPS6MV2 module is utilized to implement this service. The module automatically receives satellite signals and fixes the location. It then presents the geographical coordinates of the point of interest.

(ix) Storage

While all road contextual data are streamed on real-time basis, a 32 GB SD card is included to store the collected information for historical view and analysis. For-instance knowing the busiest road with heavy traffic flows for the entire week is very important for traffic monitoring officials. Furthermore, having knowledge about the routes that experience more air pollution saturation during peak hours is also very crucial. In most cases pollutants accumulate due to the large traffic volumes in an arterial road and therefore, redirecting some of this traffic to other routes may keep the pollution under control.

\section{(x) Received data Display}

Organic light emitting diode (OLED 128X64 COM42) display is used to demonstrate the contextual information. The display continuously presents the road name, the allowable speed limit, the weather parameters and so forth to both passengers and drivers. The OLED display is utilized as the receiver and display of the sensing data from the device components.

\section{(xi) 12 V/7 AH (20 HR) Stand-by battery}

Whereas the developed device could be powered by the bus-engine while is moving, it is supplied with a strong battery that powers $t$ when the bus is in the parking mode. The same apples to the devices deployed at the bus stops that may be at points that have no grid-power supply. 
(xii) DC-DC $24 \mathrm{~V} / 12 \mathrm{~V}$ to $5 \mathrm{~V} 3 \mathrm{~A}$ Voltage regulator

The power drawn from the battery is not suitable for the micro-controller and the mounted IoT-modules. We have used a $24 \mathrm{~V} / 15$ to $5 \mathrm{~V} 3 \mathrm{~A}$ voltage regulator to supply the micro-controller with appropriate voltage.

\subsection{Main Software Applications Used}

In this study, several specialized software applications have been employed for numerous purposes, it is therefore worthwhile very crucial to elucidate their roles in this subsection.

(i) EasyEDA

This is a web-based electronic design automation tool for various schematic designs, simulations, and printed circuit board design. It is very important to develop and evaluate our printed circuit board before pinning and mounting our sensors, and other IoT modules. EasyEDA was employed to design the printed circuit board for both the IoTmultsensing unit and the network layer gateway. It is easy to use and helps to simulate the electronic circuits.

\section{(ii) NOOBS}

Like all other computers, the raspberry Pi used for gateway development needs an operating system; it uses Linux based operating system commonly known as raspbian. It allows running both the raspbian kernel level and user level threads. NOOBS is a novel raspberry Pi firmware that makes it easy to install Raspbian operating system. Raspbian makes it possible for users to interact with the raspberry computer, through a command line interpreter (CL), or raspbian graphical user interface (GUI).

(iii) Arduino Integrated Development Environment (IDE)

This is a cross-platform software application that is used to write and upload the codes/instructions to the micro-controller boards that are compatible with arduino. The IDE is used to import all available libraries for the boards supported by different arduino versions, weather arduino Uno, arduino Mega, and arduino Nano; all functions are written in high level programming languages such as $\mathrm{C}$ and $\mathrm{C}++$.

(iv) Mydevice Cayenne IoT platform

This is a web-based IoT front-end platform that provides application programming interfaces for the end-users $t$. All sensors utilized in this article are equipped with internet connection capability specifically using WiFi technology. It is therefore possible to configure all our devices in this web-based IoT platform for convenient data retrieval and demonstration. An IoT network of devices is created and every device is allocated a unique identifier number, and a communication channel to use while it is transmitting data. For all deployed sensors, the platform avails mechanisms to set thresholds for some key parameters. For example alerting the users when the hazardous gaseous pollution reaches levels that are dangerous to human lives would serve as a stepping stone to save lives of passengers, especially those with breathing and heart related diseases.

\subsection{IoT-Based MULTI-Sensing Unit Deployment Process}

In the Figure 8, we show a simplistic procedure for the road deployment of the developed IoT based multi-sensing unit. The unit is to be deployed along the main roads from the target area. Road segmentation deployment approach is utilized based on the transmission range of the unit. If for-instance $T_{x}$ is the transmission range of the device's transmitter (in meters), for a specific road i of length $R_{L i}$ meters. The number of required deploy-able units will be determined by the number of segments created $\mathrm{N}$

$$
N=R_{L i} / T_{x}
$$




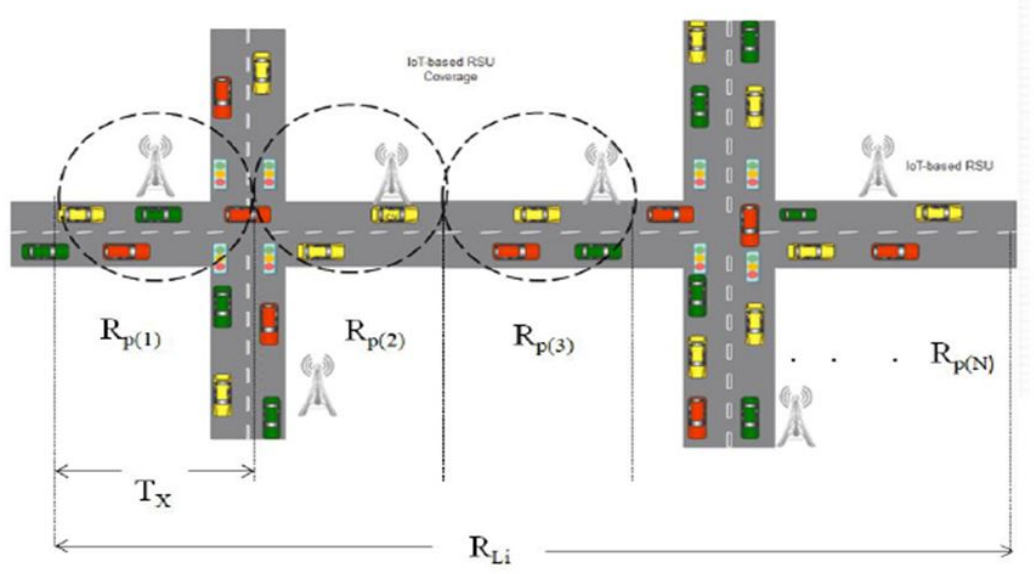

Figure 8. Device deployment process.

\subsection{Gateway Prototype for Data Transmission}

In this subsection we present an IoT-based network layer gateway prototype for numerous vehicular IoT applications. From the protocols discussed above, and available resources we have built a LoRa gateway using a raspberry $\mathrm{Pi}$ and a LoRa radio transceiver. Figure $9 \mathrm{a}, \mathrm{b}$ below show a circuit design and prototype of the LoRa gateway respectively. The developed gateway is based on three important components, the radio transceiver with its expansion board, the control unit and the power supply. RFM9x LoRa radio module is used; the module operates at 868 or $915 \mathrm{MHz}$ frequency band amateur Industrial scientific, and medical license-free frequency bands. It supports $20 \mathrm{dBm}$ LoRa radio packets, with directional antennas, the radio module could go up to $20 \mathrm{Km}$. The expansion board is constructed using a printed circuit board that interfaces the module with a raspberry Pi control unit. Another Important component of this system is its control unit that is constructed using a raspberry Pi. This is a small sized single-board computer. The raspberry Pi control unit is equipped with wireless local area network (LAN). Like other computers it has an Ethernet port as well that makes it suitable for LAN. The last but not least $\mathrm{s}$ the raspberry Pi power supply that comprises of the raspberry Pi power board with a rechargeable battery.

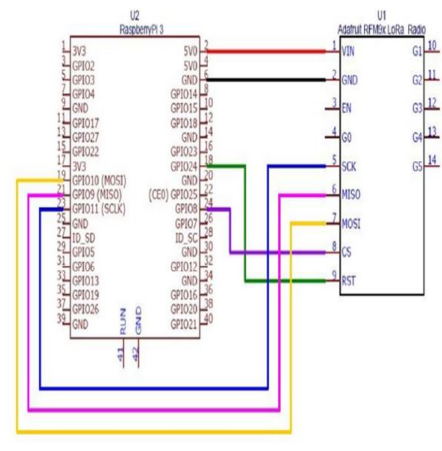

(a) Gateway circuit design

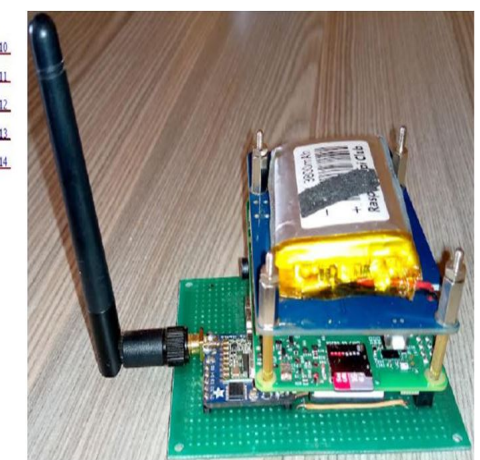

(b) Gateway Prototype

Figure 9. IoT gateway circuit design \& prototype for V-IoT. 
Two LoRa compliant sensors have been utilized to test the data transmission to the Lora-wan gateway i.e., $\mathrm{CO}_{2}$ \& PM2.5 Figure 10a,b. The sensors have been deployed on the vehicular boards along the road that is in the coverage of the gateway. Gas sensors have been used to test gateway reachable of the sensor data but other suitable sensors could now be deployed based on the user's applications.

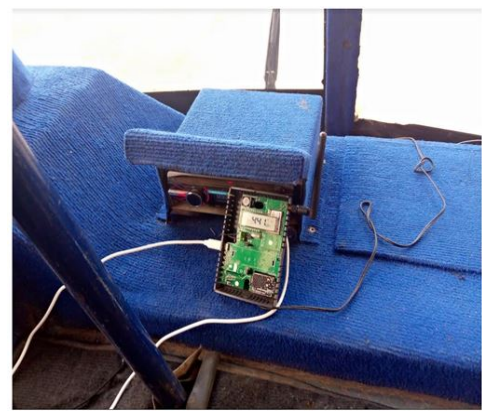

(a) $\mathrm{CO}_{2}$-Sensor: On-borad

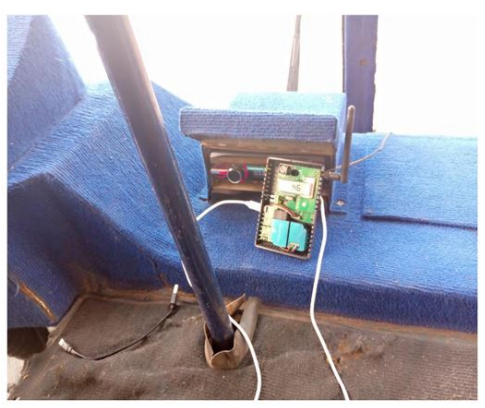

(b) PM25-sesnor: On-board

Figure 10. LoRaWAN Sensor for intra-vehicular pollution sensing.

\subsection{Sensor Data Retrieval and Triggers Algorithms from Intra-Vehicular Environments}

Algorithm 1 implements the average data retrieval and display through an intuitive web-based dashboard. Lines (2)-(3) computes the average reading for every parameter including the pollutants saturation average, temperature, humidity, and so forth. For real time reading the algorithm compares every reading with the previous reading and updates the dashboard once the readings are different Lines (5)-(8).

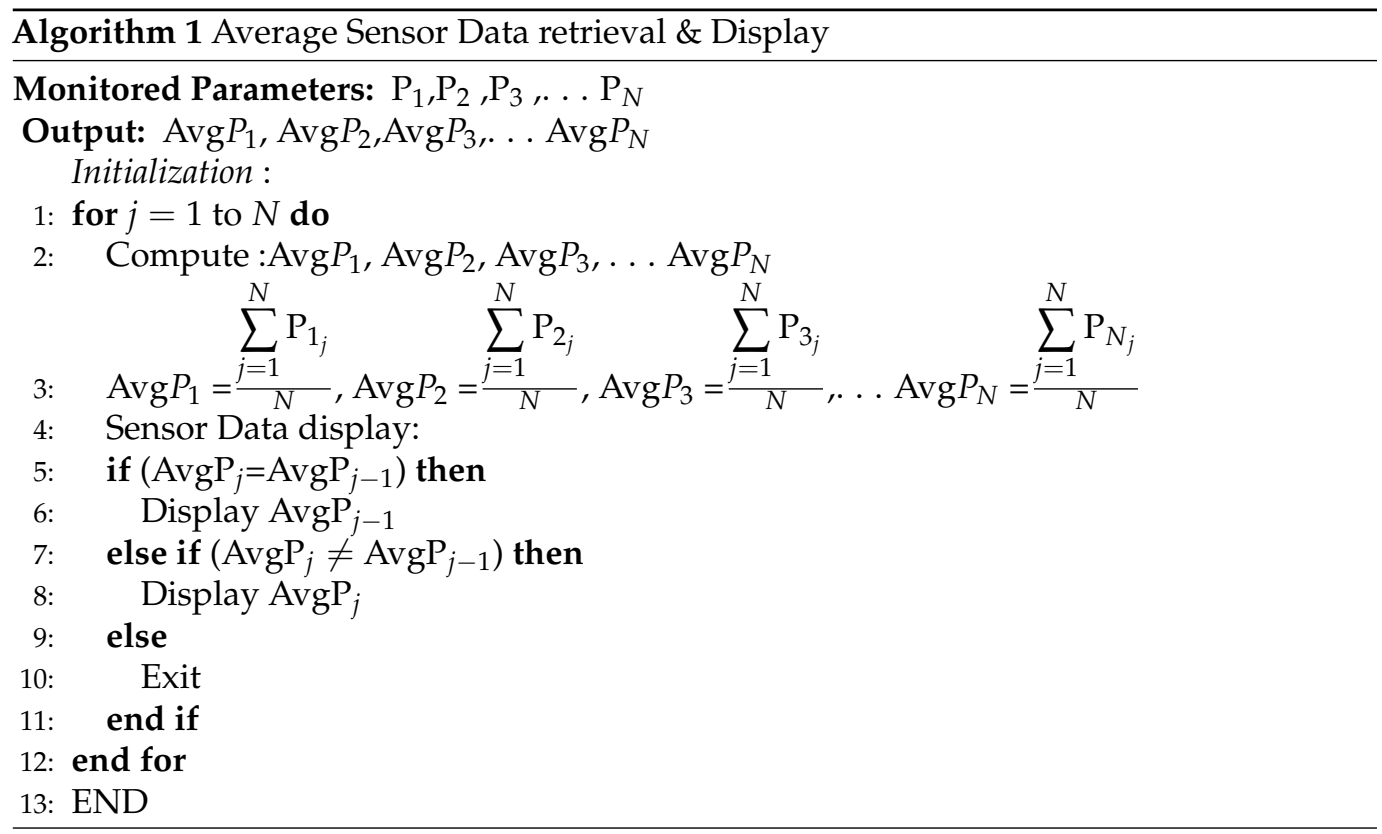

Algorithm 2 has been implemented to allow every sensing node notify the user once the monitored parameter exceeds the allowable limit. A trigger is set for every parameter under control. Though the algorithm is implemented for road contextual data collection, could be used for other smart city applications that need to alert the users. 


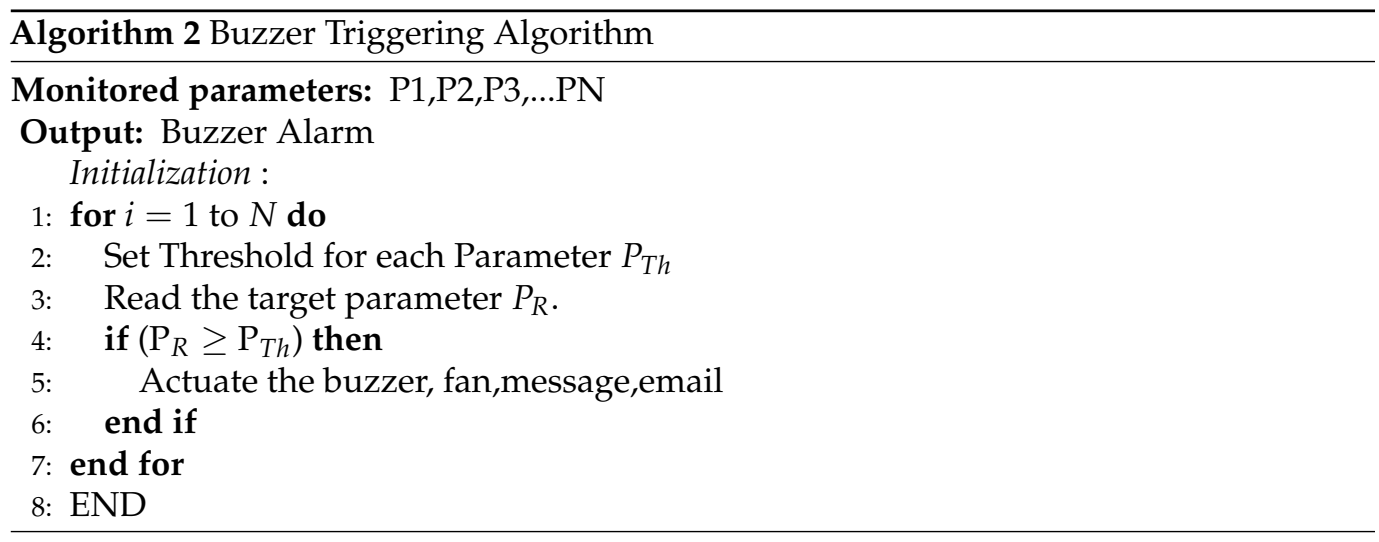

\section{On-Board Sensor Data Collection and Analysis}

One of the important services of smart city is monitoring and control of air pollution. Public buses are one of the places that gather several people at a time. It is worthwhile very crucial to monitor the level of key pollutants in public buses that have passengers on-board. Figure 11a demonstrates the daily average carbon-dioxide monitored in one of the buses from the route in the case study area. From the observation, the saturation of this pollutant increases with the number of passengers on-board. Figure $11 \mathrm{~b}$ depicts the daily average saturation of PM2.45 in the same entity. The two pollutants have been found to rise with the increased temperature. The Lora sensors employed in this study are equipped with weather measurement capacities. Temperature and humidity were measured along with the pollution saturation Figure 12a,b. Furthermore, Good communication signal is vital for data transmission in smart city services. It is therefore of great importance to keep a sharp eye on the signal status. Our system learns the communication signal status in terms of received signal strength indicator and signal to noise ratio Figure 13a,b. While the above descriptions present the historical data analyses Figures 14 and 15 demonstrates the real-time data visualization through an intuitive web-based dashboard.

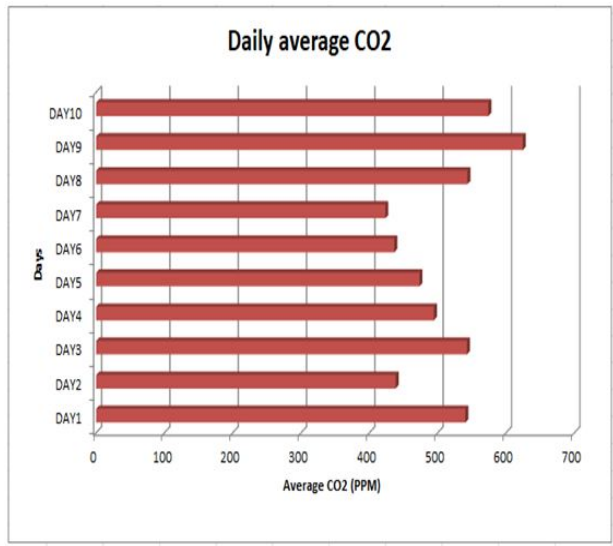

(a) On-board daily average $\mathrm{CO}_{2}$

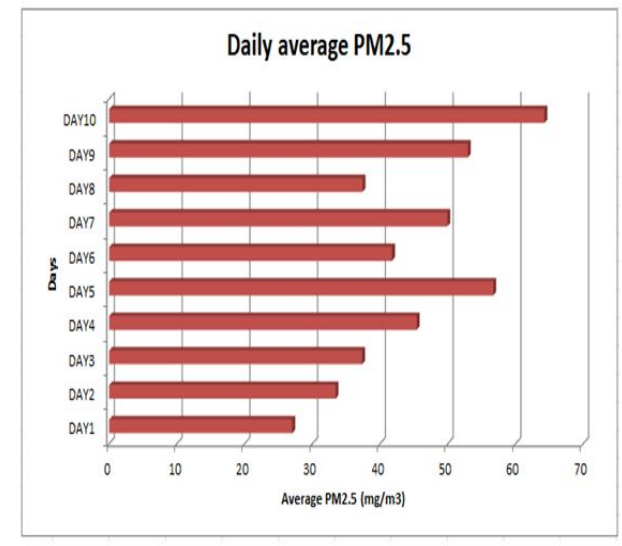

(b) On-board daily average PM2.5

Figure 11. Intra-vehicular air quality monitoring. 


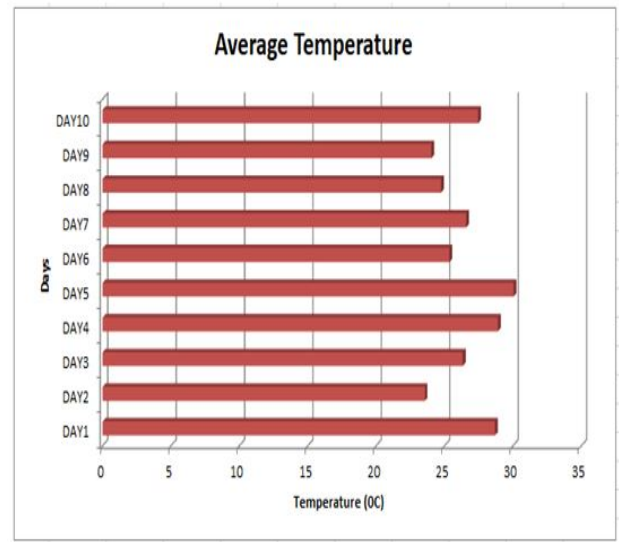

(a) On-board daily Average Temperature

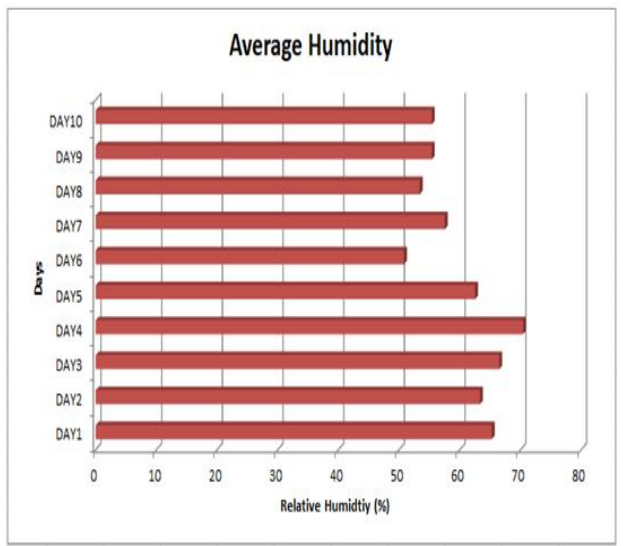

(b) On-board Average Humidity

Figure 12. Intra-vehicular Weather monitoring.

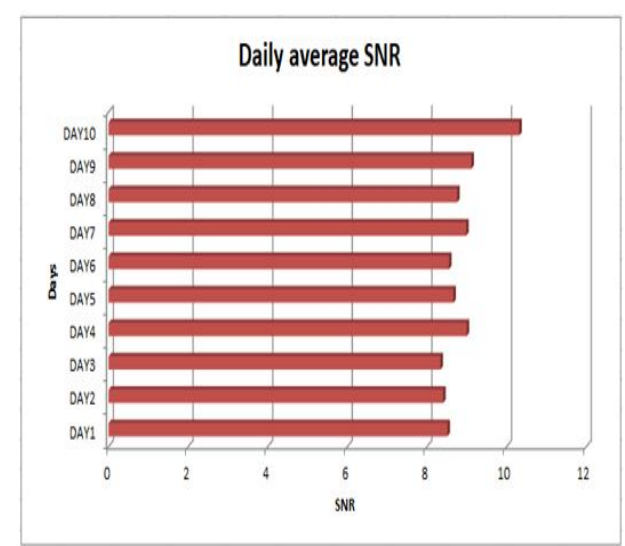

(a) On-board daily Average SNR

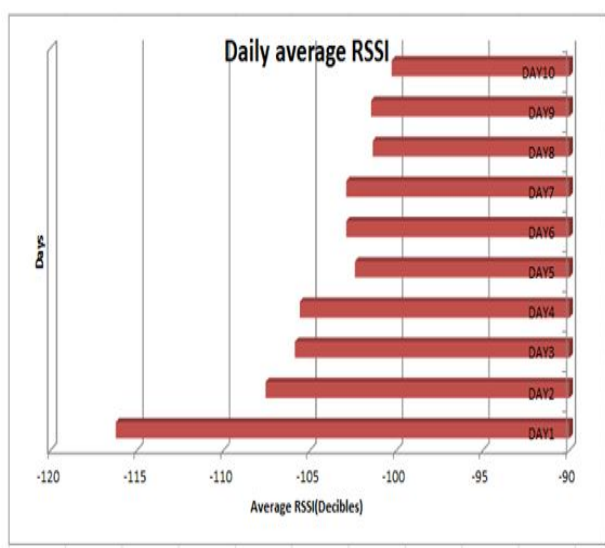

(b) On-board Average RSSi

Figure 13. Daily communication Signal Status.

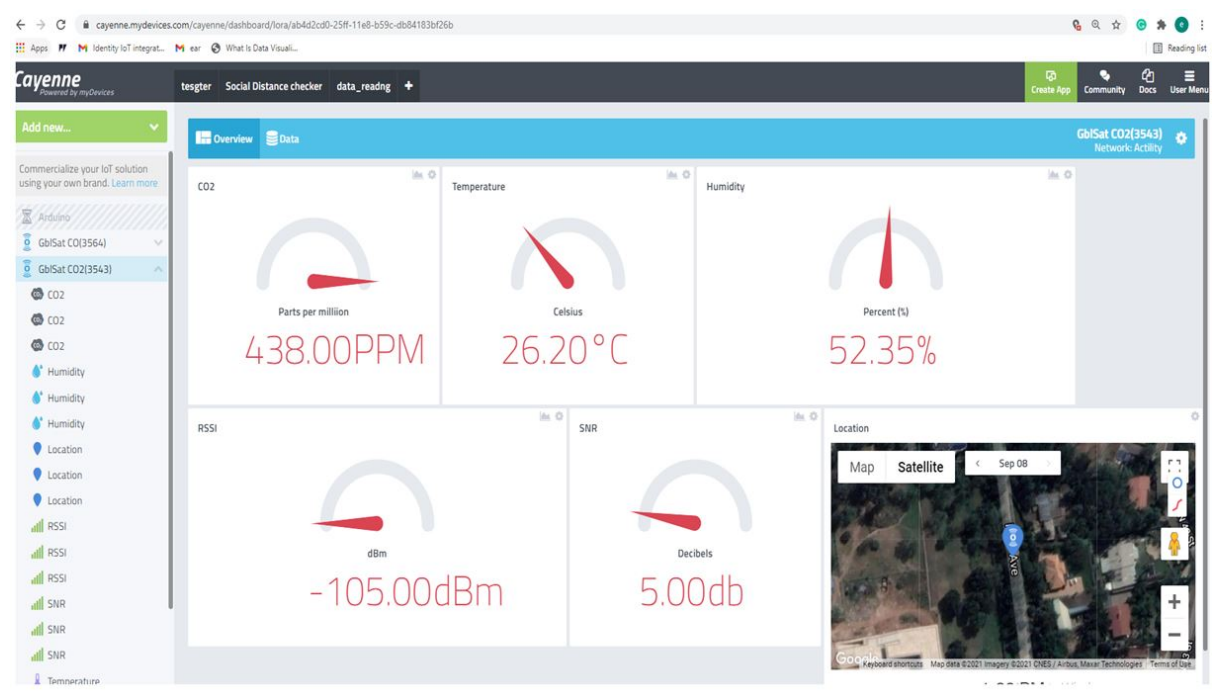

Figure 14. $\mathrm{CO}_{2}$ and Weather Real-time monitoring. 


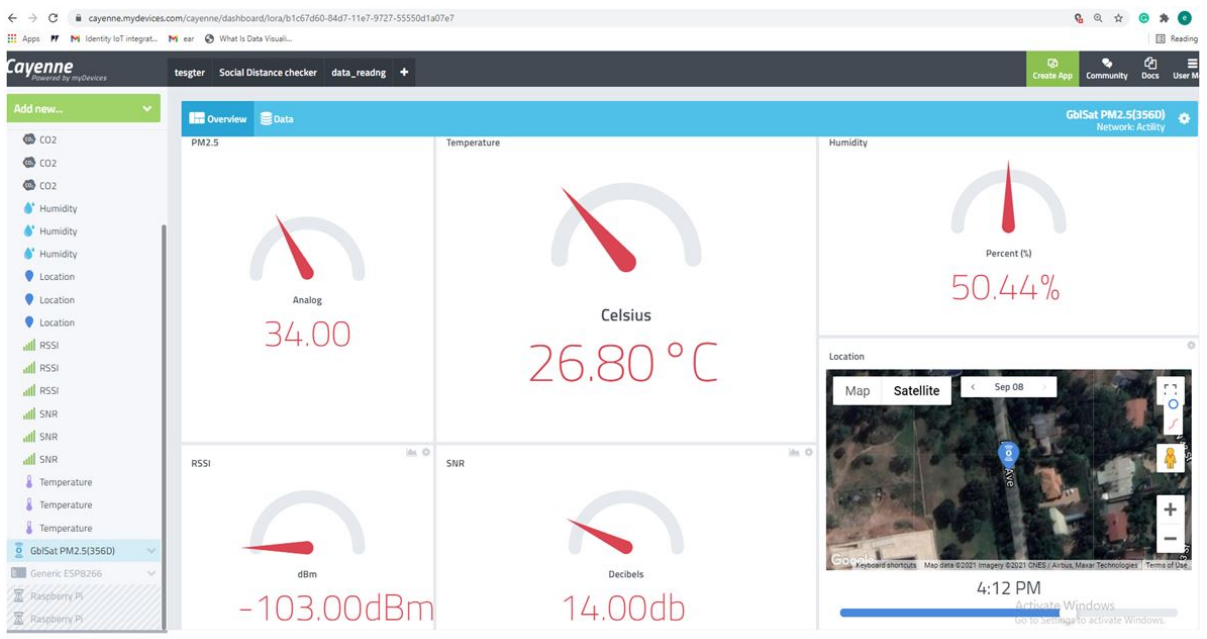

Figure 15. PM2.5 and Weather Real-time monitoring.

\section{Conclusions}

In this article, we develop and deployed different IoT systems for Vehicular use. Initially, a multi-sensing unit is designed and developed for several vehicular functions, Such as road condition monitoring, weather monitoring, vehicle position, intra-vehicular environment monitoring, and etc. A professional electronic design automation tool was used to design and evaluate the electronic circuits of all the developed IoT solutions for vehicular Internet of things. Firstly, a low cost esp32 IoT camera module with system on chip wifi is employed. The module is used for various road monitoring scenarios such as routes with heavy traffic flow volumes, road maintenance activities, road accidents and so forth. MQ135 air quality sensor was used on the same unit to keep track of a range of pollutant gases for the roads from the case study area. $\mathrm{NH}_{4}, \mathrm{CO}_{2}$, and $\mathrm{CO}$ is among the monitored gaseous pollutants. Lora sensors are deployed in the intra-vehicular environments to monitor the level of pollution. On the network layer, a loRaWAN based gateway was built and tested for use in IoT-enabled vehicular communications. Lora sensing modules on vehicular boards are able to transmit data every-time they fall into the gateway's coverage. An IoT platform was used to design the application program interface for the developed system. Data analytic and visualization is implemented using the platform. The developed solutions would enable drivers, regulators, urban transport planners, and others to take data driven decisions for a seamless transport system. Simplistic algorithms are implemented for both real-time and historical data demonstration through an intuitive web-based dashboard

Author Contributions: Idealization, Data Collection, Modeling, simulation, Device prototype and testing, Initial Draft preparation, E.T.; Application Development, and its documentation, E.T.; Draft proof-reading, co-supervision, J.R., Overall Work Supervision, R.D. All authors have read and agreed to the final draft of this manuscript.

Funding: This study was under a direct financial support of the African Center of Excellence in the Internet of Things(ACEIoT)-Under the college of Science and Technology-University of Rwanda.

Acknowledgments: The authors would like to extend their acknowledgments to the African center of excellence in the Internet of Things-University of Rwanda for the provision of required components for the prototypes.

Conflicts of Interest: The authors declare no conflict of interest.

\section{References}

1. Atzori, L.; Iera, A.; Morabito, G. The internet of things: A survey. Comput. Netw. 2010, 54, 2787-2805. [CrossRef]

2. Wortmann, F.; Flüchter, K. Internet of things. Bus. Inf. Syst. Eng. 2015, 57, 221-224. [CrossRef] 
3. Lee, I.; Lee, K. The Internet of Things (IoT): Applications, investments, and challenges for enterprises. Bus. Horiz. 2015, 58, 431-440. [CrossRef]

4. Ang, K.L.M.; Seng, J.K.P. Application specific internet of things (ASIoTs): Taxonomy, applications, use case and future directions IEEE Access 2019, 7, 56577-56590. [CrossRef]

5. Jiang, D. The construction of smart city information system based on the Internet of Things and cloud computing. Comput. Commun. 2020, 150, 158-166. [CrossRef]

6. Chu, G.; Lisitsa, A. Penetration testing for internet of things and its automation. In Proceedings of the 2018 IEEE 20th International Conference on High Performance Computing and Communications, IEEE 16th International Conference on Smart City, IEEE 4th International Conference on Data Science and Systems (HPCC/SmartCity/DSS), Exeter, UK, 28-30 June 2018; pp. 1479-1484.

7. Kaiwartya, O.; Abdullah, A.H.; Cao, Y.; Altameem, A.; Prasad, M.; Lin, C.T.; Liu, X. Internet of vehicles: Motivation, layered architecture, network model, challenges, and future aspects. IEEE Access 2016, 4, 5356-5373. [CrossRef]

8. Yang, F.; Wang, S.; Li, J.; Liu, Z.; Sun, Q. An overview of internet of vehicles. China Commun. 2014, 11, 1-15. [CrossRef]

9. Kim, T.H.; Ramos, C.; Mohammed, S. Smart City and IoT; Future Generation Computer Systems; Elsevier: Amsterdam, The Netherlands, 2017.

10. Qian, Y.; Wu, D.; Bao, W.; Lorenz, P. The internet of things for smart cities: Technologies and applications. IEEE Netw. 2019, 33, 4-5. [CrossRef]

11. Ahlgren, B.; Hidell, M.; Ngai, E.C.H. Internet of things for smart cities: Interoperability and open data. IEEE Internet Comput. 2016, 20, 52-56. [CrossRef]

12. Mehmood, Y.; Ahmad, F.; Yaqoob, I.; Adnane, A.; Imran, M.; Guizani, S. Internet-of-things-based smart cities: Recent advances and challenges. IEEE Commun. Mag. 2017, 55, 16-24. [CrossRef]

13. Reis, A.B.; Sargento, S.; Neves, F.; Tonguz, O.K. Deploying roadside units in sparse vehicular networks: What really works and what does not. IEEE Trans. Veh. Technol. 2013, 63, 2794-2806. [CrossRef]

14. Atallah, R.; Khabbaz, M.; Assi, C. Energy harvesting in vehicular networks: A contemporary survey. IEEE Wirel. Commun. 2016, 23, 70-77. [CrossRef]

15. Zhao, J.; Li, Q.; Gong, Y.; Zhang, K. Computation offloading and resource allocation for cloud assisted mobile edge computing in vehicular networks. IEEE Trans. Veh. Technol. 2019, 68, 7944-7956. [CrossRef]

16. Evariste, T.; Kasakula, W.; Rwigema, J.; Datta, R. Optimal Exploitation of On-Street Parked Vehicles as Roadside Gateways for Social IoV-A Case of Kigali City. J. Open Innov. Technol. Market Complet 2020, 6, 73. [CrossRef]

17. Abbas, M.T.; Muhammad, A.; Song, W.C. SD-IoV: SDN enabled routing for internet of vehicles in road-aware approach. J. Ambient Intell. Humaniz. Comput. 2020, 11, 1265-1280. [CrossRef]

18. Wang, X.; Zhang, R.; Gou, Y.; Liu, J.; Zhao, L.; Li, Y. Variable speed limit control method of freeway mainline in intelligent connected environment. J. Adv. Transp. 2021, 2021, 8863487. [CrossRef]

19. Krasniqi, X.; Hajrizi, E. Use of IoT technology to drive the automotive industry from connected to full autonomous vehicles. IFAC-PapersOnLine 2016, 49, 269-274. [CrossRef]

20. Philip, B.V.; Alpcan, T.; Jin, J.; Palaniswami, M. Distributed real-time IoT for autonomous vehicles. IEEE Trans. Ind. Inf. 2018, 15, 1131-1140. [CrossRef]

21. Minovski, D.; Åhlund, C.; Mitra, K. Modeling quality of IoT experience in autonomous vehicles. IEEE Internet Things J. 2020, 7, 3833-3849. [CrossRef]

22. Khayyam, H.; Javadi, B.; Jalili, M.; Jazar, R.N. Artificial intelligence and internet of things for autonomous vehicles. In Nonlinear Approaches in Engineering Applications; Springer: New York, NY, USA, 2020; pp. 39-68.

23. Guerrero-Ibanez, J.A.; Zeadally, S.; Contreras-Castillo, J. Integration challenges of intelligent transportation systems with connected vehicle, cloud computing, and internet of things technologies. IEEE Wirel. Commun. 2015, 22, 122-128. [CrossRef]

24. Guleng, S.; Wu, C.; Chen, X.; Wang, X.; Yoshinaga, T.; Ji, Y. Decentralized trust evaluation in vehicular Internet of Things. IEEE Access 2019, 7, 15980-15988. [CrossRef]

25. Rathee, G.; Jaglan, N.; Iqbal, R.; Lal, S.P.; Menon, V.G. A trust analysis scheme for vehicular networks within IoT-oriented green city. Environ. Technol. Innovat. 2020, 20, 101144. [CrossRef]

26. Reddy, M.R.; Srinivasa, K.; Reddy, B.E. Smart vehicular system based on the internet of things. J. Org. End User Comput. (JOEUC) 2018, 30, 45-62. [CrossRef]

27. $\mathrm{Du}, \mathrm{Z} . ; \mathrm{Wu}, \mathrm{C}$;; Yoshinaga, T.; Yau, K.L.A.; Ji, Y.; Li, J. Federated learning for vehicular internet of things: Recent advances and open issues. IEEE Open J. Comput. Soc. 2020, 1, 45-61. [CrossRef] 